\title{
Time to Automate the Microbial Detection and Identification: The Status Quo
}

\author{
Hafiz M.N. Iqbal' ${ }^{1 *}$ (D) and Muhammad Bilal² (iD \\ ${ }^{1}$ Tecnologico de Monterrey, School of Engineering and Sciences, Campus Monterrey, Ave. Eugenio Garza Sada \\ 2501, CP 64849 Monterrey, N.L., Mexico. ${ }^{2}$ School of Life Science and Food Engineering, Huaiyin Institute of \\ Technology, Huaian 223003, China.
}

\section{Opinion statement}

Microbiologists should closely work with policymakers, and specialists in pattern recognition, autosensing, machine learning and artificial intelligence - fast detection with high accuracy, ease in identification with less drudgery and minimal damage will result.

\section{The status quo}

The ever-increasing scientific knowledge and socio-economic awareness have drawn much concern about the emergence of new and (re)emergence of old pathogenic microbes and/or drugresistant strains with threatening concerns in different compartments of our living ecosystem (Bilal et al., 2020). For a said reason, the water contamination level due to the occurrence and persistence of bioactive ingredients or drug by-products in their active form is keep rising with the emergence of new and complex health situations (Zavala-Yoe et al., 2019; Parra-Saldivar et al., 2020). Besides the widespread distribution of pathogenic microbes and numerous biologically active drug residues/ molecules (Bilal et al., 2019), that sometimes co-exists in complex environment (Fig. 1), the overall processes efficacy, cost-effective processing, cost-benefit ratio, facile detection, identification and removal of human-health related pathogens, such as microbes are considered major hinders for the development of green planet Earth in the 21st century. So far, an array of detection and sensing measures have been developed, though using different materials, sensing cues and processing strategies. However, many of them failed to detect and identify the root cause accurately or suffers from considerable limitations. Considering the massive nanotech advancements in automation, pattern recognition, materials, machine learning, and artificial intelligence, synergistic strategies should be developed to engineer highly efficient sensing cues (Hernandez-Vargas et al., 2018).

This crucial issue should now be tackled from the root-cause by deploying the automatic detection and identification tools to assist the real-time monitoring of the generation and release of pollutants of emerging concerns, such as drug-based contaminates or drug-resistant microbes. For a proposed strategic measure, a combination of biosensors-based detection and identification could

*Correspondence: hafiz.iqbal@tec.mx

(Received: February 03, 2020; accepted: February 18, 2020)

Citation: Hafiz M.N. Iqbal and Muhammad Bilal, Time to Automate the Microbial Detection and Identification: The Status Quo, J. Pure Appl. Microbiol., 2020; 14(1):01-03. https://doi.org/10.22207/JPAM.14.1.01

(c) The Author(s) 2020. Open Access. This article is distributed under the terms of the Creative Commons Attribution 4.0 International License which permits unrestricted use, sharing, distribution, and reproduction in any medium, provided you give appropriate credit to the original author(s) and the source, provide a link to the Creative Commons license, and indicate if changes were made. 


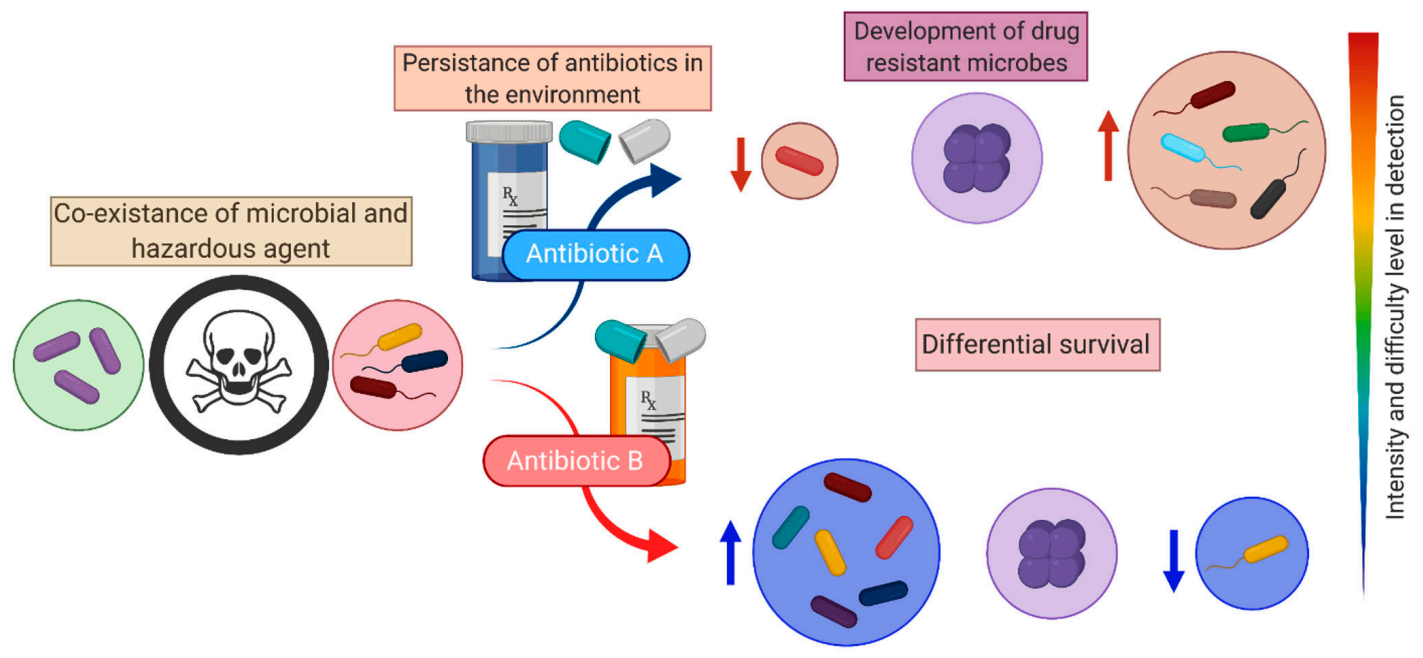

Fig. 1. A simplifies schematic illustration of the development of drug-resistant microbes due to the co-existence of microbes and hazardous agents (antibiotics) in complex environment

excellently fit as state-of-the-art tools for monitoring programs to implement legislation. Microbiologists along with taxonomists, who carefully identify and designate the microbial nomenclature, are core to the related research programs. The automated detection and identification with the aid of nanotech systems engineered by several researchers related to the autosensing, pattern recognition, machine learning, and artificial intelligence could revolutionize the taxonomy practices. This will additionally limit the strong dependency on the traditional approaches which is based on the countable expertise of a few individuals. Indeed, a rigorous interdisciplinary research and development effort, within the next decade, could lead to automated systems capable of high-throughput identifications for hundreds or thousands of types of living and/or non-living specimens. Besides the detection and identification of microbe-based agents, the real-time detection and measurement of environmentally related pollutants can also reduce the extreme consumption of numerous bioactive chemicals or reagents with an added advantage of on-site determination of contaminant composition (Hernandez-Vargas et al., 2018).

\section{Concluding remarks and outstanding questions for futuristic vision}

Though the plethora of information is available, from individual disciplines, on the exploitation of different materials and sensing cues as bio-inspired green routes for detection and identification of numerous agents. However, much of critiques, including the distribution profile of the causative agent, facile detection and safety clearance are still outstanding and need to be addressed in future studies. Furthermore, extensive scientific research with proven employability is needed in this particular line of research. Similarly, many other unsolved questions are posing a big research gap that must be tackled comprehensively.

1. Is there any significant limitation to judge the exploitation of different materials as sensing cues?

2. Can the exploitation and application of bio-sensing constructs lead some negative impact?

3. How do the detection limit and stability vary with various target agents?

4. Is there any tactic by which the problem of dispersion profile during the development of sensing cues, though using different materials and detection counterparts, can be avoided?

5. How do the size and shape-dependent efficacy of the bio-sensing constructs can be resolved? 


\section{ACKNOWLEDGMENTS}

Both listed author(s) are thankful to their representative universities/institutes for providing the related support to compile this work.

\section{CONFLICT OF INTEREST}

The authors declares that there is no conflict of interest.

\section{AUTHORS' CONTRIBUTION}

Both listed author(s) have made a substantial, direct and intellectual contribution to the work, and approved it for publication.

\section{FUNDING}

None.

\section{ETHICS STATEMENT}

This article does not contain any studies with human participants or animals performed by any of the authors.

\section{AVAILABILITY OF DATA}

Not applicable.

\section{REFERENCES}

1. Bilal, M., Ashraf, S. S., Barcelo, D., \& Iqbal, H. M. Biocatalytic degradation/redefining "removal" fate of pharmaceutically active compounds and antibiotics in the aquatic environment. Science of The Total Environment, 2019; 691: 1190-1211. https://doi. org/10.1016/j.scitotenv.2019.07.224

2. Bilal, M., Mehmood, S., Rasheed, T., \& Iqbal, H. M. Antibiotics traces in the aquatic environment: Persistence and adverse environmental impact. Current Opinion in Environmental Science \& Health, 2020; 13: 68-74. https://doi.org/10.1016/j.coesh.2019.11.005

3. Hernandez-Vargas, G., Sosa-Hernandez, J. E., Saldarriaga-Hernandez, S., Villalba-Rodriguez, A. M., Parra-Saldivar, R., \& Iqbal, H. Electrochemical biosensors: A solution to pollution detection with reference to environmental contaminants. Biosensors, 2018; 8(2): 29. https://doi.org/10.3390/bios8020029

4. Parra-Saldivar, R., Bilal, M., \& Iqbal, H. M. Life cycle assessment (LCA) in wastewater treatment technology. Current Opinion in Environmental Science \& Health, 2020; 13: 80-84. https://doi.org/10.1016/j. coesh.2019.12.003

5. Zavala-Yoe, R., Iqbal, H. M., \& Ramirez-Mendoza, R. A. Understanding the evolution of pollutants via hierarchical complexity of space-time deterministic and stochastic dynamical systems. Science of The Total Environment, 2019; 710: 136245. https://doi. org/10.1016/j.scitotenv.2019.136245 